\title{
MYC/MAX control ERK signaling and pluripotency by regulation of dual-specificity phosphatases 2 and 7
}

\author{
James Chappell, ${ }^{1}$ Yuhua Sun, ${ }^{1}$ Amar Singh, and Stephen Dalton ${ }^{2}$ \\ Department of Biochemistry and Molecular Biology, Paul D. Coverdell Center for Biomedical and Health Sciences, \\ The University of Georgia, Athens, Georgia 30602, USA
}

\begin{abstract}
Suppression of extracellular signal-regulated kinase (ERK) signaling is an absolute requirement for the maintenance of murine pluripotent stem cells (mPSCs) and requires the MYC-binding partner MAX. In this study, we define a mechanism for this by showing that MYC/MAX complexes suppress ERK activity by transcriptionally regulating two members of the dual-specificity phosphatase (DUSP) family. DUSPs function by binding and then inactivating ERK1,2 by dephosphorylating residues required for catalytic activity. MYC/MAX complexes achieve this by binding the promoters of DUSP2 and DUSP7, leading to their transcriptional activation, resulting in the attenuation of ERK activity. In the absence of MYC, ectopic DUSP2,7 expression severely delays differentiation, while loss of DUSP2,7 ectopically activates ERK, resulting in loss of pluripotency. These findings elucidate a novel regulatory role for MYC in PSC maintenance involving the stimulation of phosphatases that directly inhibit the MAPK/ERK signaling pathway. Moreover, it provides a mechanism for how leukemia inhibitory factor (LIF)/ STAT3 signaling reaches across to the MAPK/ERK pathway through MYC and MAX to sustain pluripotency.
\end{abstract}

[Keywords: MYC; pluripotent; DUSP2; DUSP7; ERK]

Supplemental material is available for this article.

Received December 18, 2012; revised version accepted March 18, 2013.

The MYC family of transcription factors comprise three family members: MYC, MYCN, and MYCL. Roles for MYC and MYCN in maintenance of pluripotency and cell reprogramming (Takahashi and Yamanaka 2006; Nakagawa et al. 2010; Araki et al. 2011) have been firmly established, although mechanisms of MYC function remain unclear. Deletion of MYC and MYCN in murine pluripotent stem cells (mPSCs) results in loss of pluripotency and differentiation toward primitive endoderm (Smith et al. 2010; Varlakhanova et al. 2010). At the molecular level, MYC maintains pluripotency by directly activating cell cycle genes and repressing genes involved in cell fate specification, such as GATA6 (Smith et al. 2010). In conjunction with its heterodimeric binding partner, MAX, MYC regulates target genes by binding to regulatory motifs known as E-boxes (5' -CACGTG-3') (Blackwell et al. 1990). Recently, MAX was shown to play an important role in maintaining PSCs by a suppressing extracellular signal-regulated kinase 1,2 (ERK1,2) activity (Hishida et al. 2011). The mechanism for how MAX regulates ERK and whether this also involves MYC is unclear, however.

\footnotetext{
${ }^{1}$ These authors contributed equally to this work.

${ }^{2}$ Corresponding author

E-mail sdalton@uga.edu

Article is online at http://www.genesdev.org/cgi/doi/10.1101/gad.211300.112
}

Regulation of MAPK/ERK is complex, and the biological outcomes of signaling through this pathway are tightly controlled by its duration, magnitude, and subcellular localization (Caunt et al. 2008). Activation of ERK by MEK-dependent phosphorylation can be countered by the activity of dual-specificity phosphatases (DUSPs) that bind and inhibit ERK activity. This phosphatase family is important for regulating spatial and temporal aspects of MAPK/ERK signaling in a wide range of cell types (Caunt et al. 2008; Patterson et al. 2009; Owens and Keyse 2007). A key requirement for mPSC self-renewal is that ERK activity be suppressed (Kunath et al. 2007). Although the mechanism by which ERK antagonizes self-renewal pathways has not been defined in mPSCs, it is clear that its activity must be maintained below a threshold level for maintenance of the pluripotent state. FGF4/ERK autocrine signaling is believed to prime PSCs for differentiation, but under self-renewal conditions, this pathway is suppressed by an as yet uncharacterized mechanism (Lanner et al. 2010). When these signaling barriers are removed, however, autocrine FGF4 signaling drives MAPK/ERK activity, resulting in differentiation (Kunath et al. 2007).

Understanding how signals converge on ERK to restrict its activity is critical for a complete understanding of how PSCs transition to a differentiated state. The observation 
that MAX restricts ERK activity in PSCs (Hishida et al. 2011) offers a potential answer to this general question, but it remains unclear how MAX blocks full ERK activation. In this study, we show that MYC and MAX transcriptionally regulate two members of the DUSP family that serve to suppress ERK activity (Dowd et al. 1998; Theodosiou and Ashworth 2002). These findings establish critical new roles for MYC and MAX in pluripotency through regulation of the ERK pathway and establish a broader signaling network linking leukemia inhibitory factor (LIF)/STAT3 to MAPK/ERK.

\section{Results}

MYC suppresses ERK activity in murine pluripotent cells

Since previous studies established that loss of MAX leads to increased ERK activity and loss of pluripotency (Hishida et al. 2011), we asked whether this was also true following loss of MYC and MYCN. This was tested using a mPSC line carrying floxed alleles of $M Y C$ and $M Y C N$ (Smith et al. 2010) and a transgene expressing a Cre recombinaseglucocorticoid receptor fusion (CreGR). Addition of the glucocorticoid dexamethasone (Dex) translocates the CreGR fusion to the nucleus, where it efficiently deletes floxed MYC and MYCN alleles, as indicated by the loss of MYC and MYCN protein within $24 \mathrm{~h}$ (Fig. 1A). This coincided with the activation of ERK; up-regulation of endoderm transcript markers such as GATA6, GATA4, SOX17, and SOX7; and loss of alkaline phosphatase (AP) activity (Fig. 1A-C). All of these responses were blocked by addition of the MEK inhibitor UO126. We also independently confirmed that loss of MAX increases ERK activity (Supplemental Fig. 1). Ectopic expression of a MYC-estrogen receptor fusion protein (MYC-ER) under control of the estrogen analog 4-hydroxytamoxifen (4OHT) blocked the activation of ERK in MYC- and MYCN-deleted cells (Fig. 1D) and maintained GATA6 transcripts at levels comparable with the wild-type control (Fig. 1E). These data indicate that MYC and MAX maintain pluripotent cells by suppressing ERK activity. This provides an explanation for how mPSCs can be maintained in MEK/GSK3 inhibitor (2i)-based media where MYC expression is reduced (Ying et al. 2008; Marks et al. 2012).

\section{MYC regulates transcription of DUSP2 and DUSP7}

To explain these observations mechanistically, we searched for MYC target genes in the MYC Cancer Gene: MYC Target Gene Database (http://www.myccancergene.org/site/ myctargetdb.asp) that could potentially regulate ERK activity. Direct regulation of ERK by MYC was ruled out because the ERK1,2 protein remained constant irrespective of MYC status (Fig. 1A). Of the known MYC target genes that can be potentially regulated by MYC, the most notable were members of the DUSP gene family (DUSP2/ 7). Consistent with this possibility, the DUSP7 protein is down-regulated in parallel to MYC and MYCN during murine embryonic stem cell (mESC) differentiation, just prior to the activation of ERK (Fig. 2A). We note a delay in the activation of ERK following the decline of DUSP7 levels; this suggests that low levels of DUSP continue to inhibit ERK during the early stages of differentiation. Along with OCT4 and NANOG, DUSP2/7 transcripts also decline following LIF withdrawal, while FGF5 transcript levels increase (Fig. 2B). DUSP7 protein is made under all conditions tested (FCS/LIF, 2i, and BPM4+LIF), whereas DUSP2 and another protein implicated in ERK regulation, DUSP9 (Li et al. 2012), show condition-specific expression patterns (Supplemental Fig. 2).

To investigate whether MYC regulates DUSP2/7 transcript levels, $M Y C$ and MYCN were deleted by addition of Dex to the double-floxed MYC and MYCN PSCs. Consistent with this possibility, loss of MYC and MYCN led to decreased levels of DUSP2/7 mRNA (Fig. 2C). A second experiment was then performed in which MYC activity was further elevated in wild-type R1 ESCs using an inducible MYC-ER transgene. This showed that addition of $4 \mathrm{OHT}$ further elevated DUSP2/7 transcripts. Results in Figure 2, C and D, therefore indicate that MYC regulates DUSP2/7. To examine this in further detail, we asked whether DUSP2/7 could be reactivated in cells that lost expression due to $M Y C$ deletion. This involved $M Y C$ deletion (+Dex) for $3 \mathrm{~d}$ and ectopic MYC-ER expression from day 2 onward. This experiment shows that following loss of endogenous MYC and MYCN, DUSP2/7 mRNA levels decrease, but this can be rescued by ectopic MYC expression (MYC-ER+4OHT) (Fig. 2E). These results indicate that MYC regulates DUSP2/7 in PSCs.

The possibility that DUSP2/7 are directly regulated by MYC/MAX was suggested by the presence of E-boxes in their respective promoter regions (Fig. 2F,G). To establish whether MYC/MAX regulate DUSP2/7 directly, we first performed chromatin immunoprecipitation (ChIP) analysis on the promoter regions of both genes. This analysis showed significant enrichment of MAX, MYC, and MYCN binding in proximal promoter regions for the two genes (Fig. 2F,G). In addition to this, ectopic expression of MYC-ER (+4OHT) increased DUSP2/7 promoter activity above already elevated levels, as shown by luciferase reporter assays (Supplemental Fig. 3), and loss of MYC activity significantly reduced MYC, MYCN, and MAX binding to the DUSP2,7 promoters (Supplemental Fig. 4). These data further support the model that MYC/MAX regulates DUSP2,7 at the level of transcription and that down-regulation of MYC underpins decreased DUSP expression and ERK activation during differentiation.

\section{DUSP2/7 are required for MYC-dependent suppression of $E R K$}

The data presented so far point to a mechanism in which MYC promotes pluripotency by suppressing ERK activity through a DUSP2/7-dependent mechanism. To establish whether DUSP7 activity is sufficient to suppress ERK activation in PSCs, we generated a MYC and MYCN floxed ESC line expressing DUSP7 under the control of a tetracycline (Tet)-inducible promoter (Fig. 3A). In the presence of endogenous MYC and MYCN (-CreGFP), ERK activity and differentiation markers GATA6, GATA4, 


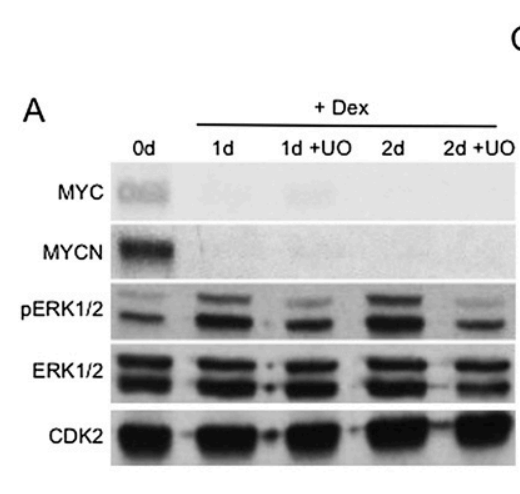

C
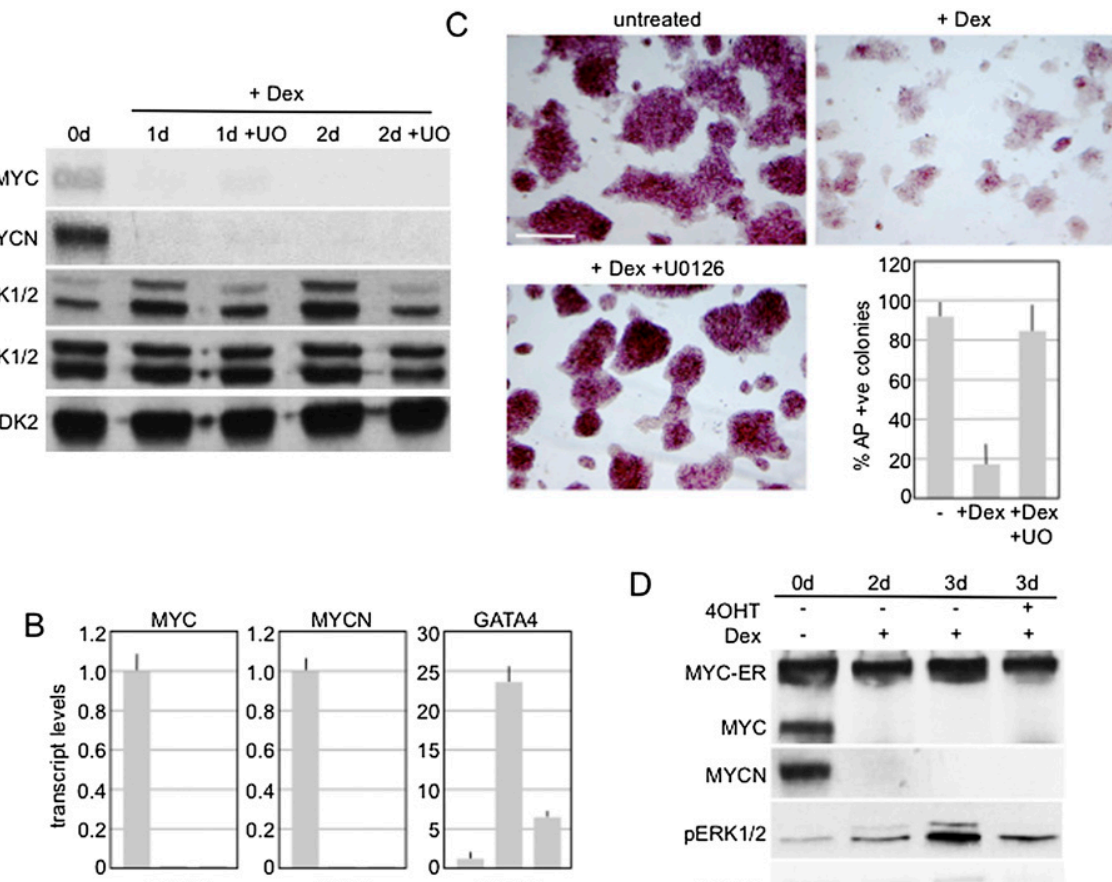

D
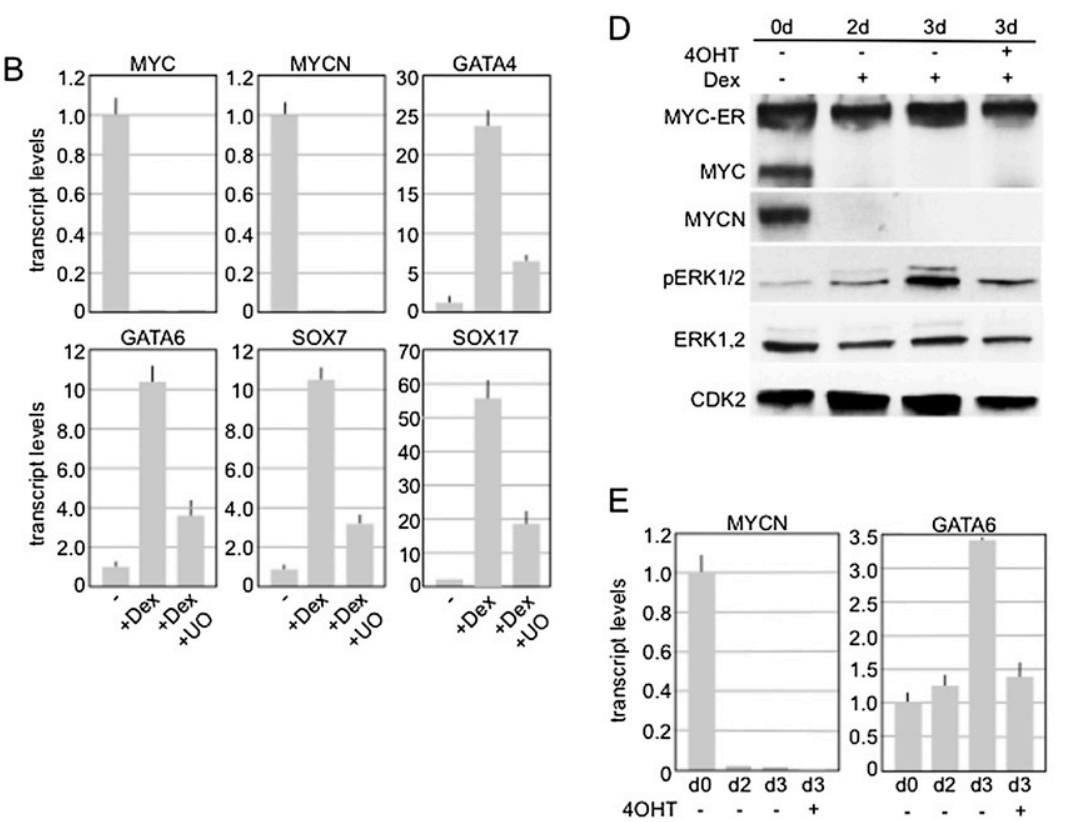

Figure 1. MYC regulates ERK activity in mPSCs. (A) Whole-cell lysates from $\mathrm{MYC}^{\mathrm{fl} / \mathrm{fl}} \mathrm{MYCN}^{\mathrm{fl} / \mathrm{fl}} \mathrm{CreGR}^{\mathrm{f}}$ cells grown without treatment or with Dex for $1 \mathrm{~d}$ and $2 \mathrm{~d} \pm \mathrm{U} 0126(\mathrm{UO})$ were immunoblotted and probed with antibodies as indicated. $(B)$ Expression of MYC and primitive endoderm marker transcripts were assayed from $\mathrm{MYC}^{\mathrm{fl} / \mathrm{fl}} \mathrm{MYCN}^{\mathrm{fl} / \mathrm{fl}}$ CreGR cells grown $\pm 2 \mathrm{~d}$ of Dex treatment \pm UO126. Fold changes were normalized to $\mathrm{MYC}^{\mathrm{fl} / \mathrm{fl}} \mathrm{MYCN}^{\mathrm{fl} / \mathrm{fl}} \mathrm{CreGR}$ cells that were not exposed to Dex. Error bars represent standard deviation. $(C) \mathrm{MYC}^{\mathrm{fl} / \mathrm{fl}} \mathrm{MYCN}^{\mathrm{fl} / \mathrm{fl}}$ CreGR cells grown $\pm 2 \mathrm{~d}$ of Dex treatment \pm UO126 were stained for AP activity and then scored as being $\mathrm{AP}^{+}$or $\mathrm{AP}^{-}(N=195)$. Bar, $100 \mu \mathrm{m}$. $(D)$ Whole-cell lysates from $\mathrm{MYC}^{\mathrm{fl} l / \mathrm{fl}} \mathrm{MYCN}^{\mathrm{fl} l / f l}$ CreGR MYC-ER cells grown with no treatment and with Dex for $2 \mathrm{~d}, 3 \mathrm{~d}$, and $3 \mathrm{~d}+24 \mathrm{~h} 4 \mathrm{OHT}$ were immunoblotted and probed with antibodies as indicated. $(E)$ Expression of the indicated transcripts was determined from the conditions in $D$, and fold changes were normalized to the "no treatment" condition.

and SOX17 remain low but are elevated following loss of MYC and MYCN due to CreGFP expression (Fig. 3B,C). Tet-induced expression of DUSP7, however, completely blocked the up-regulation of phospho-ERK and GATA6, GATA4, and SOX17 transcripts, indicating that DUSP7 is a major effector of MYC-dependent MAPK signaling in PSCs.

We then asked whether DUSP7 could maintain characteristics of pluripotent cells following LIF withdrawal. Within $4 \mathrm{~d}$ of LIF withdrawal, the percentage of APpositive colonies dropped from almost $90 \%$ to $<2 \%$. In contrast, enforced expression of DUSP7 following addition of Tet maintained the percentage of AP-positive colonies at $\sim 35 \%$ (Fig. 3D,E). Together, these results show that DUSP7 can suppress ERK activation in the absence of MYC and can partially substitute for LIF in promoting pluripotency.

\section{DUSP2 and DUSP7 are required for maintenance of pluripotency}

To establish whether DUSP2,7 are required for PSC maintenance, we transduced mESCs with lentiviral constructs expressing shRNAs that knocked down DUSP2 by $>80 \%$ and DUSP7 by $>90 \%$ (Fig. 4A). Transduction of both lentiviruses caused a distinct morphology change to 
Chappell et al.

A

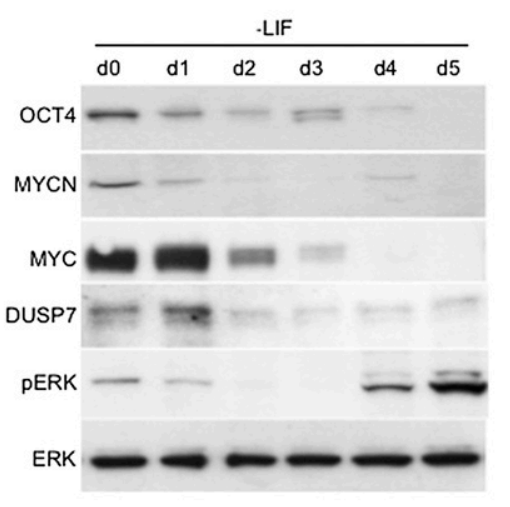

C $\frac{\text { MYC MYCN deletion }}{\text { DUSP2 } \square \text { DUSP7 }} \frac{\text { MYC-ER: ectopic MYC }}{\text { DUSP2 } \square \text { DUSP7 }}$
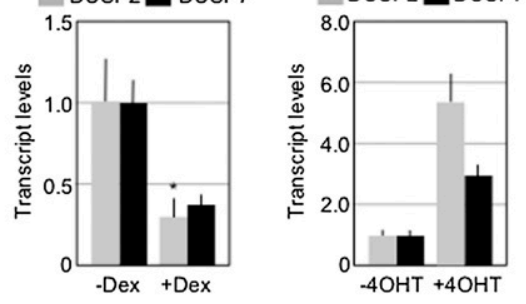

F

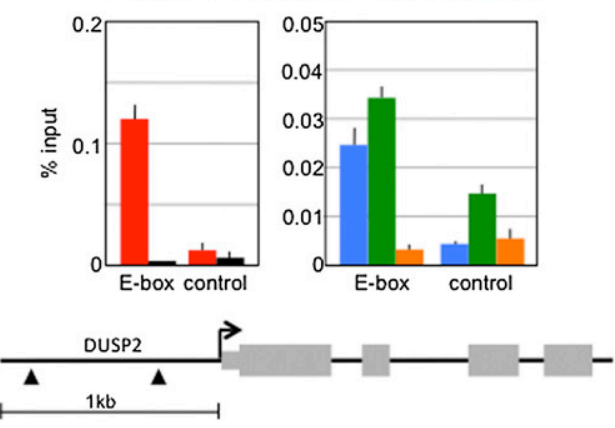

B
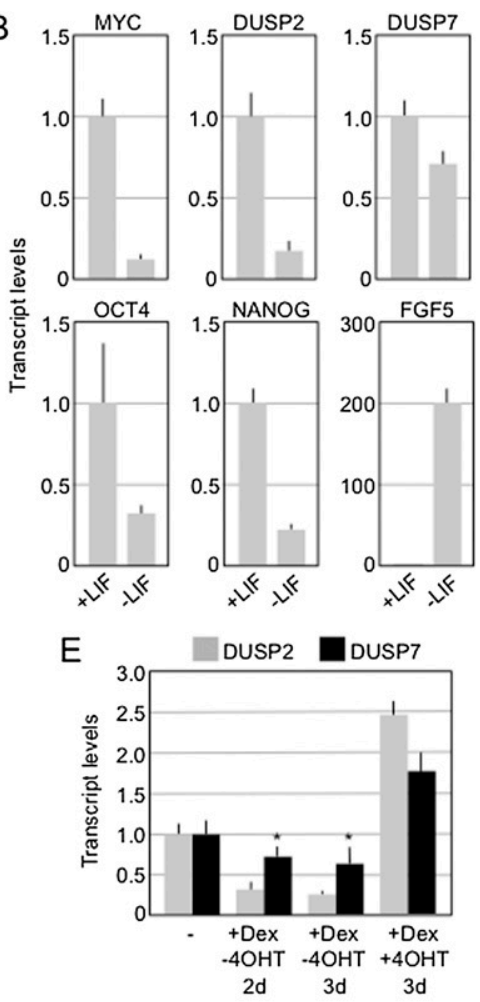

G $\square$ MAX $\square$ IgG $\square$ MYC $\square$ MYCN $\square \lg$

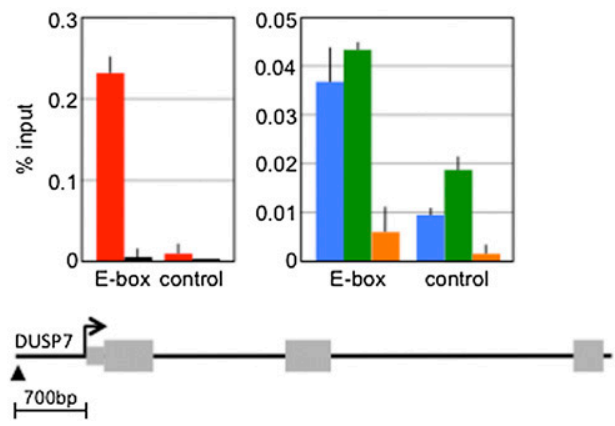

Figure 2. MYC transcriptionally regulates DUSP2 and DUSP7. (A) Whole-cell lysates from R1 mESCs grown in differentiating conditions (-LIF) were collected over $5 \mathrm{~d}$ in $24 \mathrm{~h}$ increments and then immunoblotted and probed with antibodies as indicated. $(B)$ Transcript levels were assayed in R1 ESCs and $4 \mathrm{~d}$ following withdrawal of LIF. Fold changes in transcript levels were determined in triplicate after normalization to untreated cells. Error bars represent standard deviation. (C) DUSP2 and DUSP7 transcript levels were assayed in $\mathrm{MYC}^{\mathrm{fl} / \mathrm{fl}} \mathrm{MYCN}^{\mathrm{fl} / \mathrm{fl}}$ - or $\mathrm{MYC}^{-/-} \mathrm{MYCN}^{-/-}$-deleted cells. CreGR expression was induced by addition of Dex (+Dex) for $48 \mathrm{~h}$. $(D)$ Wild-type R1 mESCs carrying a MYC-ER transgene were induced by addition of $4 \mathrm{OHT}$ for $24 \mathrm{~h}$. Transcript levels were assayed as in $C$. (E) DUSP2 and DUSP7 transcript levels were assayed from $\mathrm{MYC}^{\mathrm{fl} / \mathrm{fl}} \mathrm{MYCN}^{\mathrm{fl} / \mathrm{fl}}$ CreGR MYC-ER cells without or after Dex treatment for $2 \mathrm{~d}, 3 \mathrm{~d}$, and $3 \mathrm{~d}+24 \mathrm{~h} 4 \mathrm{OHT}$. Fold changes were normalized to untreated cells. $(F, G)$ MYC/MAX specifically bind to the promoter region of DUSP2 $(F)$ and DUSP7 $(G)$. ChIP-immunoprecipitated DNA was amplified by quantitative PCR (qPCR) with primers spanning the DUSP2,7 promoters. Schematic representations of DUSP2,7 are shown with potential MYC-binding sites (E-boxes) indicated by triangles. All E-boxes are canonical with the sequence $5^{\prime}$-CACGTG- $\left.3^{\prime} . .^{\star}\right) P<0.05$. All other $P$-values are $<0.01$. "Control" regions are located $2 \mathrm{~kb}$ upstream of the transcription start sites of DUSP2 and DUSP7 and do not contain an E-box.

mESC colonies, indicative of differentiation (Fig. 4B). This was associated with increased ERK activity, a reduction in NANOG protein (Fig. 4C), and up-regulation of the endoderm marker FOXA2 (Fig. 4D). Similar effects were observed with different shRNAs targeting DUSP2 and DUSP7 (Supplemental Fig. 5), indicating that the effects were specific to knockdown of DUSPs. To test the requirement for DUSP2/7 in pluripotency, LacZ ${ }^{+}$mESCs were transduced with DUSP2 and DUSP7 shRNAs and then injected into blastocyst stage embryos to evaluate embryonic contribution. Thirteen days after the introduction of blastocysts into recipient females, embryos were evaluated for developmental contribution by control (GFP) or DUSP2,7 shRNA transduced mESCs (Fig. 4E). LacZ $^{+}$cells transduced with GFP shRNA lentivirus broadly contributed to embryonic tissues $(67 \%)$, whereas 

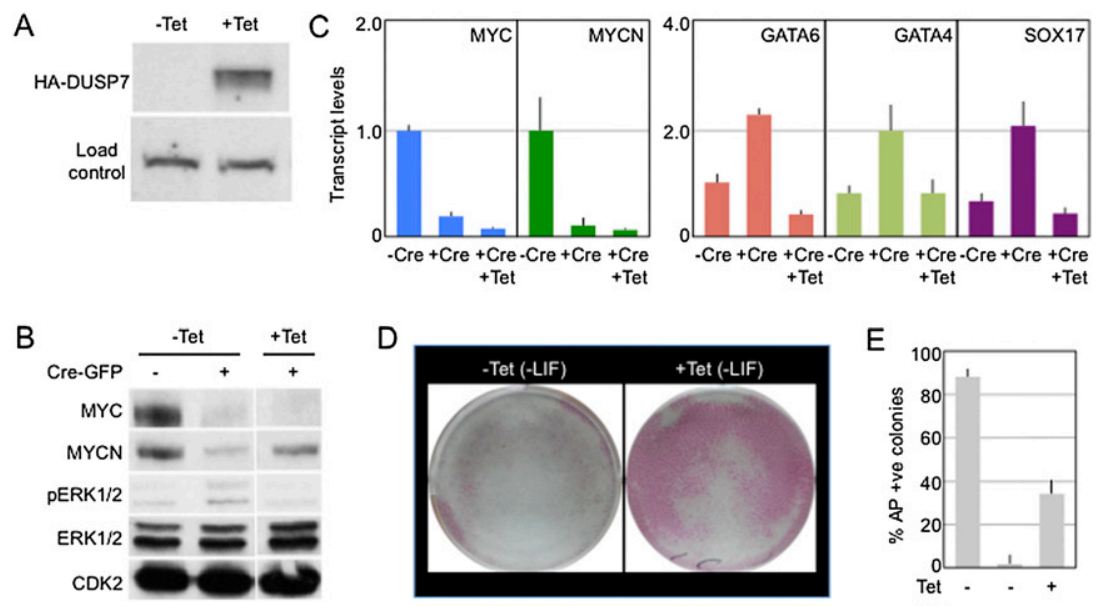

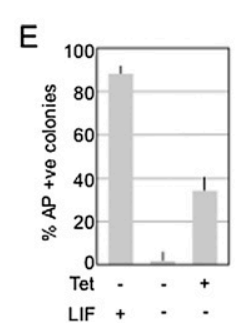

Figure 3. Down-regulation of MYC, MYCN, DUSP2, and DUSP7 correspond to an increase in ERK activity and differentiation. (A) Tet regulation of HA-DUSP7 ESC line grown in the absence $(-)$ or presence $(+)$ of Tet activator protein. After $24 \mathrm{~h}$ induction (+Tet), cells were harvested and subjected to immunoblot analysis, probing with HA monoclonal antibody to detect DUSP7. Load control, HA cross-reacting, nonspecific protein was used as a load control. (B) $\mathrm{MYC}^{\mathrm{fl} / \mathrm{fl}} \mathrm{MYCN}^{\mathrm{f} / / \mathrm{fl}}$ cells containing Tet-inducible DUSP7 were transfected with a CreGFP vector \pm Tet and then sorted for GFP $48 \mathrm{~h}$ post-transfection. Whole-cell lysates were then immunoblotted and probed with antibodies as indicated. $(C)$ Transcript levels from $B$ were assayed in triplicate. Fold changes were normalized to untreated $\mathrm{MYC}^{\mathrm{fl} / \mathrm{fl}} \mathrm{MYCN}^{\mathrm{fl} / \mathrm{ll}}$ cells. Error bars represent standard deviation. $(D)$ Tet-inducible DUSP7 ESCs were plated on 24-well plates. After LIF withdrawal, Tet-express was added to induce DUSP7 expression. Cells were allowed to grow in differentiating condition for $4 \mathrm{~d} \pm$ Tet express and then stained for AP activity. (E) The percentage of AP-positive colonies under each condition is shown. All assays were performed in triplicate $(N=164)$.

DUSP2 and DUSP7 knockdown cells did so at a greatly reduced frequency $(22 \%)$. Overall, these data show that DUSP2/7 are required for maintenance of pluripotency.

\section{Binding of DUSP7 to ERK is required for self-renewal of pluripotent cells}

Commercially available antibodies for DUSP7 do not immunoprecipitate its target efficiently, so we transfected cells with a construct expressing epitope (HA)-tagged DUSP7 to investigate interactions between DUSP and ERK. Reciprocal immunoprecipitations between DUSP7 and ERK in whole-cell lysates showed specific interactions (Fig. 5A,B), confirming that DUSP7 interacts with ERK in mESCs. To evaluate the role of DUSP7 binding to ERK further, we expressed a Tet-regulated hypermorphic allele of $E R K 2(\mathrm{D} 319 \mathrm{~N})$ that is defective in binding DUSP proteins (Chu et al. 1996). Under conditions that normally promote mESC self-renewal (+LIF), expression of $E R K 2^{\mathrm{D} 319 \mathrm{~N}}$ following addition of Tet resulted in reduction of NANOG protein (Fig. 5B) and a significant reduction in the percentage of AP-positive colonies (Fig. 5C,D). Together, these data indicate that ERK becomes activated in pluripotent cells when it is refractory to inhibition by DUSP7. Under these conditions, PSC self-renewal is significantly compromised.

\section{Discussion}

A recent study describing MYC as a general transcriptional amplifier in mice indicates that it serves as a global regulator of active genes (Nie et al. 2012). Our data are not inconsistent with these findings that some of its functions in self-renewal can be substituted for by only a small number of genes. Deletion of MYC and MYCN in mESCs promotes endoderm differentiation (Smith et al. 2010), but this can be abrogated by ectopically expressing one of its key targets, DUSP7. Taking this into account, it is intriguing that mESCs can be maintained with significantly reduced MYC levels under conditions where ERK signaling is inhibited (Hishida et al. 2011; Marks et al. 2012). It seems that ERK signaling also negatively impacts reprogramming because the MEK inhibitor PD035901 enhances induced PSC (iPSC) generation efficiency (Lin et al. 2009). This observation predicts that MYC could be substituted by MEK inhibitor or DUSP knockdown in repro-gramming cocktails.

\section{MYC suppresses ERK activity via DUSP2/7}

Evidence supporting the significance of MYC and MAX in PSC biology has rapidly accumulated, but its important role in regulation of ERK activity has not been previously characterized at the mechanistic level. The mechanisms associated with pluripotency and/or self-renewal in which MYC has been implicated are diverse and include promotion of rapid cell cycle progression, increased rates of metabolism and cell growth, and direct repression of important lineage-specifying genes. In this study, we determined a previously unidentified role for MYC and MAX where together they maintain pluripotency by suppressing ERK through a DUSP-dependent mechanism (Fig. 6).

LIF is generally considered to be a pluripotency maintenance factor through its ability to bind LIF receptor/ gp130 heterodimers by transducing signals through the activation of STAT3 (Niwa et al. 1998). A second aspect of LIF signaling involves the gp130-dependent phosphorylation of SHP-2 that activates ERK and, paradoxically, serves to antagonize pluripotency maintenance networks (Burdon et al. 1999). An important target of STAT3 in PSCs is $M Y C$, a transcription factor with known but poorly defined roles in the maintenance of PSCs /Cartwright et al. 2005). In this study, we propose a model in which LIF controls ERK activity by using MYC as a downstream 
Chappell et al.
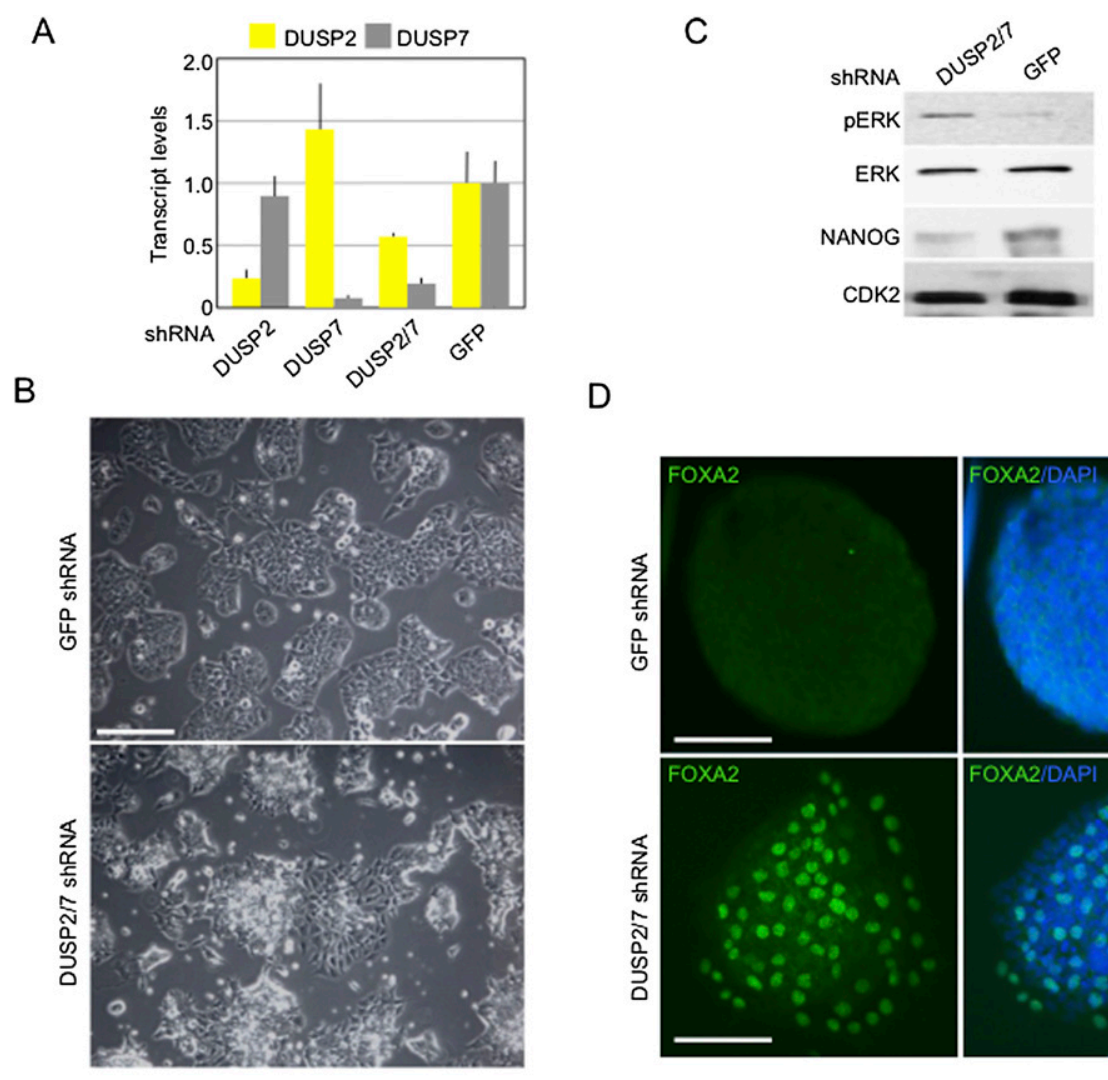

$\mathrm{D}$
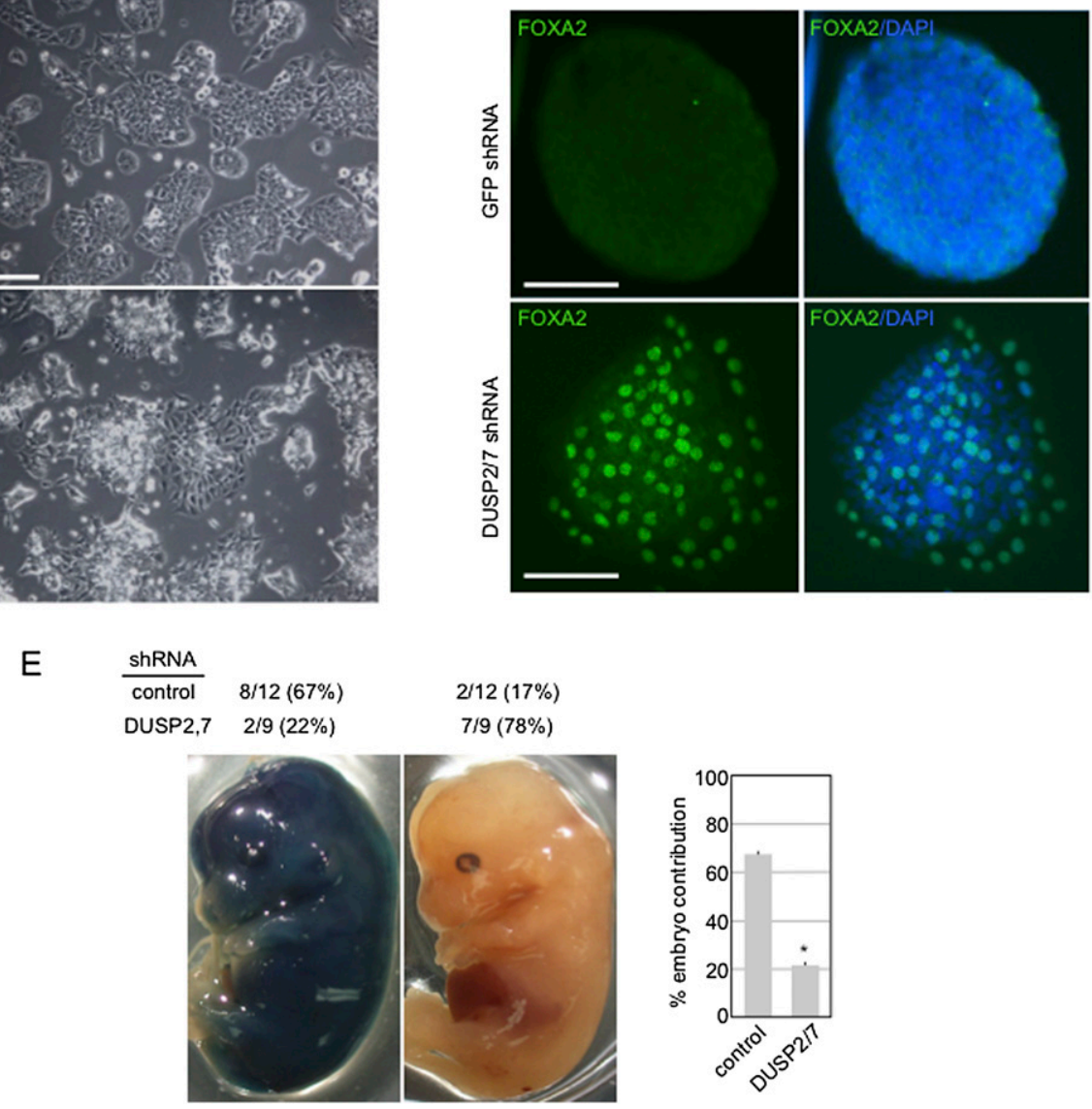

Figure 4. DUSP2/7 expression is necessary for maintenance of mPSCs. (A) R1 mESCs were infected with lentiviruses expressing shRNAs for DUSP2 (TRCN0000028960), DUSP7 (TRCN0000080728), DUSP2 and DUSP7, or GFP (control). Fours days after lentiviral transduction, target knockdown was assayed in triplicate by qPCR. Fold changes in transcripts were determined after normalization to GFP knockdown cells. Error bars represent standard deviation. (B) Colony morphology after DUSP2/7 and GFP knockdown (4 d). Bar, $100 \mu \mathrm{m}$. (C) Whole-cell lysates were collected after DUSP2/7 and GFP shRNA knockdown. Samples were immunoblotted and probed with antibodies as indicated. $(D)$ Immunofluorescent staining using FOXA2 antibody after DUSP2/7 and GFP knockdown. Bar, $50 \mu \mathrm{m}$. (E) R1 mESCs marked by $\beta$-galactosidase activity were infected with lentiviruses expressing shRNAs for DUSP2/7 or GFP control and injected into blastocyst stage C57BL/6 embryos. After transfer into recipient females, embryos were allowed to develop until embryonic day 14.5 (E14.5). LacZ staining was then performed on fixed, whole embryos. The number of blastocysts injected, the number of chimeras generated, and the percentage of chimeras generated are indicated (control, $N=21$; DUSP2/7, $N=18$ ). The percentage of embryo contribution for control and DUSP2/7 knockdown cells is graphed on the right. $\left.{ }^{*}\right) P<0.05$.

effector to activate DUSP transcription (see Fig. 6). An unexpected outcome of this is the realization that LIF/ gp130 has the potential to simultaneously activate and represses ERK activity through separate signaling pathways. In the context of self-renewal, its role in activation of the STAT3/MYC/DUSP pathway is critical. In the scenario where LIF is withdrawn, MYC levels decrease, leading to loss of DUSP activity. In the absence of restraining signals, FGF4-dependent autocrine signals are then free to elevate ERK activity, and differentiation 
A

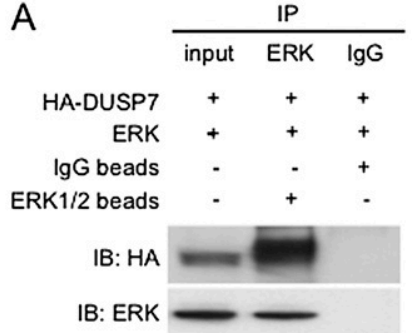

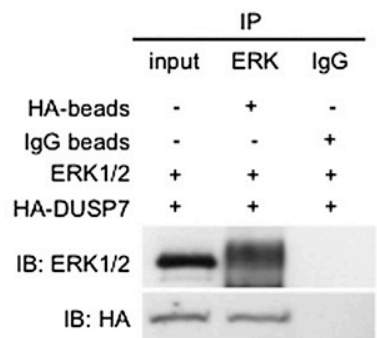

B

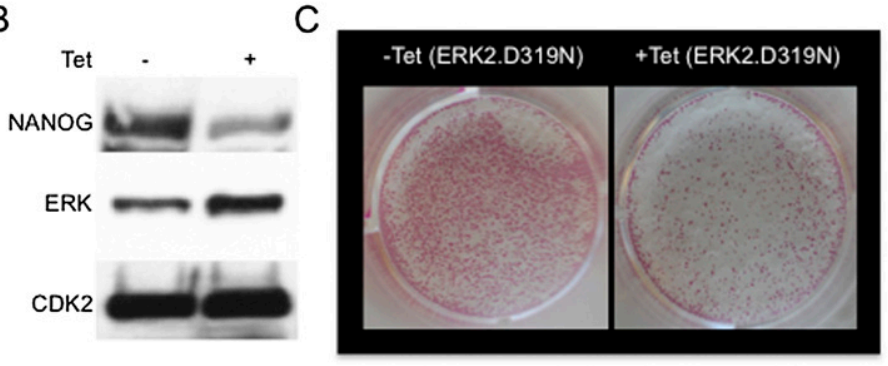

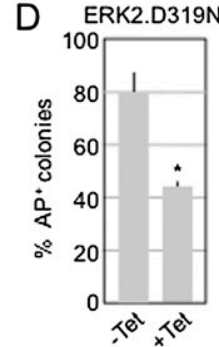

Figure 5. DUSP7 directly interacts with ERK in PSCs. (A) HA-DUSP7-expressing ESC line was established by transfecting a Tet-inducible HA-DUSP7 expression plasmid into R1 ESCs. After $1 \mathrm{~d}$ of Tetexpress induction, cell lysate was made for coimmunoprecipitation (IP) experiments. Reciprocal immunoprecipitation of DUSP7 and ERK was detected using ERK1/2 and HA antibodies. (B) R1 mESCs containing Tetinducible ERK2(D319N) were cultured \pm Tet for $2 \mathrm{~d}$. Whole-cell lysates were then immunoblotted and probed with antibodies as indicated. (C) Tet-inducible ERK2(D319N) cells were cultured \pm Tet for $2 \mathrm{~d}$, followed by -Tet for $3 \mathrm{~d}$, and then stained for AP. (D) The percentage of APpositive colonies under each condition was determined from triplicate experiments. Error bars represent standard deviation $(N=74) .\left({ }^{\star}\right) P<0.05$. All other $P$-values are $<0.01$. then follows (Lanner et al. 2010). It is unlikely that DUSPs are the only class of phosphatases that have the capacity to dephosphorylate ERK. In other cell types and in other signaling scenarios, DUSP-independent modulation of ERK by protein phosphatases are likely to be important.

\section{ERK suppression in different signaling contexts}

The experiments that we present here were performed in traditional media containing fetal calf serum supplemented with LIF. Under these conditions, MYC, MYCN, DUSP2, and DUSP7 are expressed at high levels. Another study recently reported that in LIF media supplemented with BMP4, self-renewal was promoted by Smad-dependent transcriptional activation of DUSP9 (Li et al. 2012). Under these conditions, the BMP4-SMAD and LIFSTAT3-MYC pathways synergistically cosuppress ERK activity by promoting DUSP9 and DUSP2/7 expression, respectively. This indicates that multiple signaling inputs can simultaneously converge on ERK to suppress its activity. DUSP7 was the only DUSP to be robustly expressed under a wide range of conditions, including LIF/FCS, LIF/BMP, and 2i. DUSP9 expression, for example, was restricted to $\mathrm{BMP} / \mathrm{LIF}$ conditions and is therefore unlikely to play a major role in ERK regulation in $2 \mathrm{i}$ or traditional LIF/FCS conditions.

In total, the results presented provide new insight into how the LIF-STAT3 signaling pathway promotes selfrenewal. An important aspect of this mechanism involves new roles for MYC in the modulation of MAPK/ ERK signaling through DUSP effector molecules. Although different DUSP family members may collaborate and be regulated themselves by different signaling pathways, DUSP7 seems to be the most broadly used under a range of culture conditions. Our data, together with the previously known role of ERK in regulation of MYC (Yeh et al. 2004), suggest that a positive feedback loop may exist where MYC stimulates its own activity through regulation of DUSP. This is the first study showing a role for MYC in the modulation of cell signaling - a mechanism that has clear implications for its role in tumor development. It will be interesting to establish whether MYC/MAX regulate DUSP activity in other cell types or whether this regulation is restricted to PSCs.

\section{Materials and methods}

\section{Cell culture and blastocyst injections}

R1 mESCs, $M Y C^{f 1 / f 1} M Y C N^{f 1 / f 1}$ miPSCs (Smith et al. 2010), $M A X^{-/-}$mESCs (Hishida et al. 2011), and LacZ ${ }^{+}$miPSCs (Smith

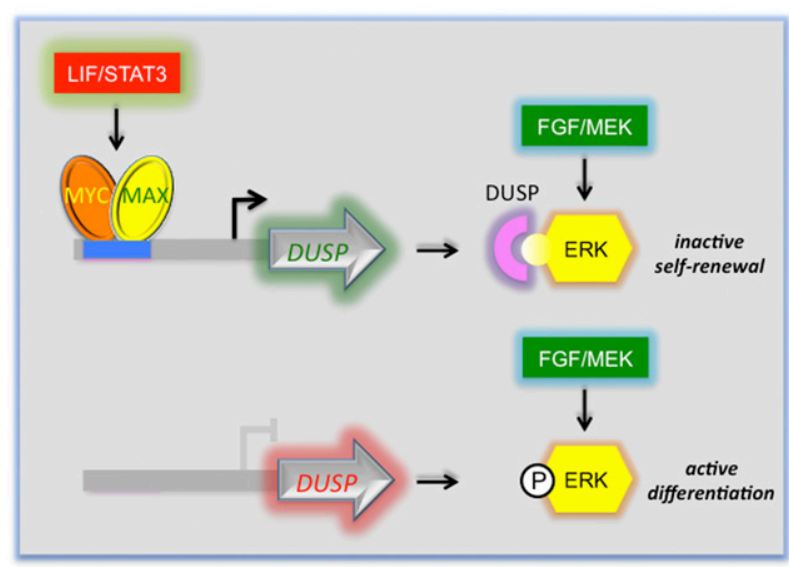

Figure 6. A model for ERK regulation by MYC/MAX complexes in murine pluripotent cells. MYC/MAX activates transcription of DUSP2 and DUSP7 in their E-box-containing promoters. The protein products are characterized by phosphatase activity necessary for dephosphorylation of phospho-ERK. Suppressing ERK activity is necessary for maintenance of a pluripotent state and is necessary in the presence of autocrine FGF signaling. 
et al. 2010) were cultured in LIF and fetal calf serum-supplemented medium on gelatin-coated dishes. The following reagents were used at the concentrations indicated: Dex $(20 \mu \mathrm{M})$, U0126 (20 $\mu \mathrm{M})$, doxycycline (1 $\mu \mathrm{g} / \mathrm{mL})$, and 4OHT (100 nM). The BMP4/LIF culture condition was used as described previously (Ying et al. 2003). The 2i culture condition was used as described previously (Ying et al. 2008). Differentiation was performed in attachment or suspension culture in medium lacking LIF or knockout serum replacement (KSR). Blastocyst injections and embryo analysis were performed as described previously (Cartwright et al. 2005).

\section{Lentiviral transduction for shRNA knockdown}

The shRNA plasmids for DUSP2 (TRCN0000028961), DUSP7 (TRCN0000080729), and the GFP control (RHS4459) were purchased from Open Biosystems. To make lentivirus, shRNA plasmids and Trans-lenti shRNA packaging plasmids were transfected into H293T cells according to the kit manual (Open Biosystems, TLP4615). After determining the virus titer, mESCs were transduced at a multiplicity of infection of 5:1. Puromycin selection $(1 \mu \mathrm{g} / \mathrm{mL})$ for $4 \mathrm{~d}$ was applied to select cells with stable viral integration. Quantitative PCR (qPCR) and Western blot were used to assess the knockdown of targets.

\section{Tet-inducible expression}

DUSP2-HA, DUSP7-HA, and ERK2(D319N) fragments were cloned from cDNA clones (Origene) and placed under the control of a Tet-inducible promoter from pLVX-tight-puro (Clontech, PT3996-5). In the absence of Tet-express, pLVX-tight-puro provides very low background expression, whereas addition of Tet-express strongly transactivates target genes. Expression constructs were transfected into mESCs using Lipofectamine 2000 and stable cell lines established with $1 \mathrm{mg} / \mu \mathrm{L}$ puromycin selection. To induce target gene expression, $3 \times 10^{5}$ Tet-inducible cells were plated in six-well plates. The next day, Tet-expresstransducible protein (Clontech, \#631178) was added for $1 \mathrm{~h}$ in serum-free medium to induce gene expression. Cells were allowed to grow in complete medium for an additional $12-24 \mathrm{~h}$ before assay for gene induction.

\section{Transcript expression assays and luciferase assays}

RNA was isolated with the RNeasy minikit (Qiagen). cDNA libraries were prepared from RNA using the iScript reverse transcription kit (Bio-Rad). Transcript levels were assayed by RT-qPCR using TaqMan assays (Applied Biosystems) as described by Smith et al. (2010). Transcript levels were normalized to GAPDH and performed in triplicate. Luciferase assays were performed with the Dual Luciferase Reporter kit (Promega) according to instructions and analyzed on a Synergy 2 plate reader (BioTek). Unless stated, $P$-values relating to changes in transcript levels were $<0.01$.

\section{Immunoblotting, immunostaining, and ChIP}

For immunoblotting, immunostaining, and ChIP experiments, we used commercially available antibodies to MYC (Santa Cruz Biotechnology, sc-764), phospho-ERK1/2 (Cell Signaling, 9102), ERK1/2 (Cell Signaling, 9102), MYCN (Calbiochem, OP13), MAX (Santa Cruz Biotechnology, sc-197), OCT4 (Santa Cruz Biotechnology, sc-5279), DUSP7 (Santa Cruz Biotechnology, sc-47667), ERK1/2 (Santa Cruz Biotechnology, sc-135900), NANOG (CosmoBio, REC-RCAB0002PF), rabbit IGG (Abcam, ab46540), and FOXA2 (Millipore, 07-633). The following primer sets for ChIP-qPCR were designed using Primer3 (Rozen and
Skaletsky 2000): DUSP2 promoter F (5'-GGACAATTTCCTG ACGGTTG-3') and R (5'-CCATTTTCTCCCAGGGTTTT-3'), DUSP2 control F (5'-AACCATGTCTAGGGGTGTGC-3') and $\mathrm{R}\left(5^{\prime}\right.$-CAGCACCAATTACAGCGAGA-3'), DUSP7 promoter $\mathrm{F}\left(5^{\prime}\right.$ GCCTAACACAGAGCACGACA-3') and R (5'-AGAGCCAGTC TTCCCTCCTC-3'), and DUSP7 control F (5'-GGACTGAGG GCTTGAGAGTG-3') and R (5'-CTTAGCAAGCAGCACGT GAG-3'). AP staining was carried out with an AP staining kit (Sigma). Immunoblotting, immunostaining, AP staining, and ChIP experiments were as described previously (Smith et al. 2010).

\section{Acknowledgments}

This work was supported by grants to S.D. from the National Institute of Child Health and Human Development (HD049647) and the National Institute for General Medical Sciences (GM75334).

\section{References}

Araki R, Hoki Y, Uda M, Nakamura M, Jincho Y, Tamura C, Sunayama M, Ando S, Sugiura M, Yoshida MA, et al. 2011. Crucial role of c-Myc in the generation of induced pluripotent stem cells. Stem Cells 29: 1362-1370.

Blackwell TK, Kretzner L, Blackwood EM, Eisenman RN, Weintraub H. 1990. Sequence-specific DNA binding by the c-Myc protein. Science 250: 1149-1151.

Burdon T, Stracey C, Chambers I, Nichols J, Smith A. 1999. Suppression of SHP-2 and ERK signalling promotes self-renewal of mouse embryonic stem cells. Dev Biol 210: 30-43.

Cartwright P, McLean C, Sheppard A, Rivett D, Jones K, Dalton S. 2005. LIF/STAT3 controls ES cell self-renewal and pluripotency by a Myc-dependent mechanism. Development 132: $885-896$.

Caunt CJ, Rivers CA, Conway-Campbell BL, Norman MR, McArdle CA. 2008. Epidermal growth factor receptor and protein kinase C signaling to ERK2: Spatiotemporal regulation of ERK2 by dual specificity phosphatases. I Biol Chem 283: 6241-6252.

Chu Y, Solski PA, Khosravi-Far R, Der CJ, Kelly K. 1996. The mitogen-activated protein kinase phosphatases PAC1, MKP-1, and MKP-2 have unique substrate specificities and reduced activity in vivo toward the ERK2 sevenmaker mutation. J Biol Chem 271: 6497-6501.

Dowd S, Sneddon AA, Keyse SM. 1998. Isolation of the human genes encoding the pyst 1 and Pyst 2 phosphatases: Characterisation of Pyst2 as a cytosolic dual-specificity MAP kinase phosphatase and its catalytic activation by both MAP and SAP kinases. J Cell Sci 111: 3389-3399.

Hishida T, Nozaki Y, Nakachi Y, Mizuno Y, Okazaki Y, Ema M, Takahashi S, Nishimoto M, Okuda A. 2011. Indefinite selfrenewal of ESCs through Myc/Max transcriptional complexindependent mechanisms. Cell Stem Cell 9: 37-49.

Kunath T, Saba-El-Leil MK, Almousailleakh M, Wray J, Meloche S, Smith A. 2007. FGF stimulation of the Erk1/2 signalling cascade triggers transition of pluripotent embryonic stem cells from self-renewal to lineage commitment. Development 134: 2895-2902.

Lanner F, Lee KL, Sohl M, Holmborn K, Yang H, Wilbertz J, Poellinger L, Rossant J, Farnebo F. 2010. Heparan sulfationdependent fibroblast growth factor signaling maintains embryonic stem cells primed for differentiation in a heterogeneous state. Stem Cells 28: 191-200.

Li Z, Fei T, Zhang J, Zhu G, Wang L, Lu D, Chi X, Teng Y, Hou N, Yang $\mathrm{X}$, et al. 2012. BMP4 signaling acts via dual-specificity 
phosphatase 9 to control ERK activity in mouse embryonic stem cells. Cell Stem Cell 10: 171-182.

Lin T, Ambasudhan R, Yuan X, Li W, Hilcove S, Abujarour R, Lin X, Hahm HS, Hao E, Hayek A, et al. 2009. A chemical platform for improved induction of human iPSCs. Nat Methods 6: $805-808$.

Marks H, Kalkan T, Menafra R, Denissov S, Jones K, Hofemeister H, Nichols J, Kranz A, Stewart AF, Smith A, et al. 2012. The transcriptional and epigenomic foundations of ground state pluripotency. Cell 149: 590-604.

Nakagawa M, Takizawa N, Narita M, Ichisaka T, Yamanaka S. 2010. Promotion of direct reprogramming by transformationdeficient Myc. Proc Natl Acad Sci 107: 14152-14157.

Nie Z, Hu G, Wei G, Cui K, Yamane A, Resch W, Wang R, Green DR, Tessarollo L, Casellas R, et al. 2012. c-Myc is a universal amplifier of expressed genes in lymphocytes and embryonic stem cells. Cell 151: 68-79.

Niwa H, Burdon T, Chambers I, Smith A. 1998. Self-renewal of pluripotent embryonic stem cells is mediated via activation of STAT3. Genes Dev 12: 2048-2060.

Owens DM, Keyse SM. 2007. Differential regulation of MAP kinase signalling by dual-specificity protein phosphatases. Oncogene 26: 3203-3213.

Patterson KI, Brummer T, O'Brien PM, Daly RJ. 2009. Dualspecificity phosphatases: Critical regulators with diverse cellular targets. Biochem I 418: 475-489.

Rozen S, Skaletsky H. 2000. Primer3 on the WWW for general users and for biologist programmers. Methods Mol Biol 132: 365-386.

Smith KN, Singh AM, Dalton S. 2010. Myc represses primitive endoderm differentiation in pluripotent stem cells. Cell Stem Cell 7: 343-354.

Takahashi K, Yamanaka S. 2006. Induction of pluripotent stem cells from mouse embryonic and adult fibroblast cultures by defined factors. Cell 126: 663-676.

Theodosiou A, Ashworth A. 2002. MAP kinase phosphatases. Genome Biol 3: reviews3009-reviews3009.10.

Varlakhanova NV, Cotterman RF, deVries WN, Morgan J, Donahue LR, Murray S, Knowles BB, Knoepfler PS. 2010. myc maintains embryonic stem cell pluripotency and selfrenewal. Differentiation 80: 9-19.

Yeh E, Cunningham M, Arnold H, Chasse D, Monteith T, Ivaldi G, Hahn WC, Stukenberg PT, Shenolikar S, Uchida T, et al. 2004. A signalling pathway controlling c-Myc degradation that impacts oncogenic transformation of human cells. Nat Cell Biol 6: 308-318.

Ying QL, Nichols J, Chambers I, Smith A. 2003. BMP induction of Id proteins suppresses differentiation and sustains embryonic stem cell self-renewal in collaboration with STAT3. Cell 115: 281-292.

Ying QL, Wray J, Nichols J, Batlle-Morera L, Doble B, Woodgett J, Cohen P, Smith A. 2008. The ground state of embryonic stem cell self-renewal. Nature 453: 519-523. 


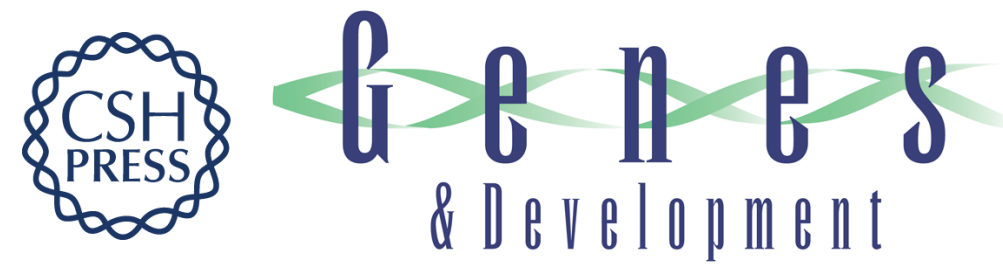

\section{MYC/MAX control ERK signaling and pluripotency by regulation of dual-specificity phosphatases 2 and 7}

James Chappell, Yuhua Sun, Amar Singh, et al.

Genes Dev. 2013, 27:

Access the most recent version at doi:10.1101/gad.211300.112

Supplemental
Material http://genesdev.cshlp.org/content/suppl/2013/04/16/27.7.725.DC1

References This article cites 26 articles, 9 of which can be accessed free at: http://genesdev.cshlp.org/content/27/7/725.full.html\#ref-list-1

License

Email Alerting Receive free email alerts when new articles cite this article - sign up in the box at the top Service right corner of the article or click here.

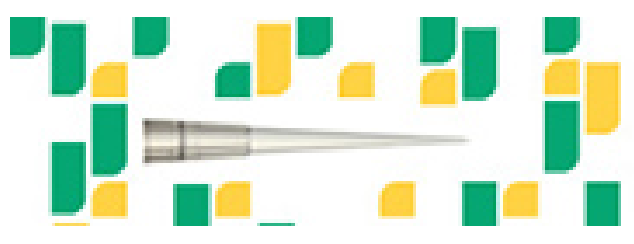

Focused on your science. 